\title{
Thoracic X-Strap Harness. Design and Method 3
}

\section{Volen Arkumarev}

This design and method has been used to attach tags to Egyptian Vultures, Eurasian Griffon Vultures Gyps fulvus, Imperial Eagles Aquila heliaca and White-tailed Eagles in Bulgaria and Greece.

\section{Equipment needed:}

- Teflon ribbon: $11.2 \mathrm{~mm} / 0.44$ " wide (Griffon, Cinereous); $8.4 \mathrm{~mm} / 0.33$ " wide (Egyptian)

- Ornitela device, Microwave Telemetry, E-obs and Ecotone

- Scissors

- Clamp scissors

- Needles (1 mm/1.2 mm)

- Needle threader (according to the size of the needle)

- Curved needle (1 mm/1.2 mm)

- Kevlar fishing thread $(0.35 \mathrm{~mm}$ or $0.30 \mathrm{~mm})$

- Superglue (Loctite glue)

- Paint marker pen - silver

- Ruler

- Neoprene pad

- Neoprene glue

\section{Attachment must be prepared in advance.}
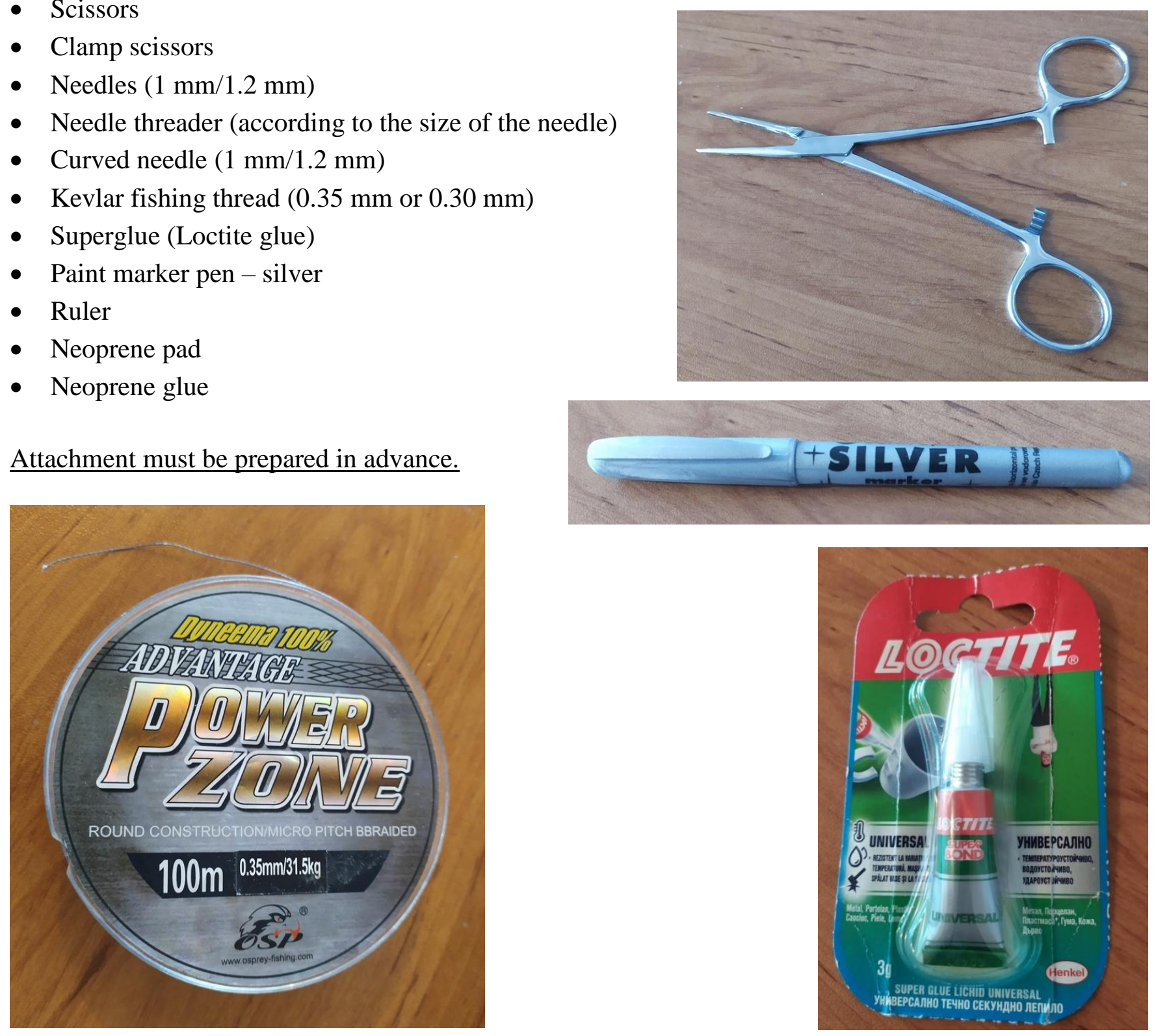

Figure 3.1: Equipment. 


\section{Measurements and preparations: Key stages/steps}

Need $120 \mathrm{~cm}$ length of Teflon ribbon.

1) There are two initial preparation methods, depending on the type of device used:

a) If the device has two attachment lugs at the front and two at the back, cut the ribbon into four $30 \mathrm{~cm}$ lengths. Take one piece of $30 \mathrm{~cm}$ Teflon ribbon and feed the ribbon through one of the attachment lugs just enough to tie both ends into a knot. Tie a simple knot to secure the ribbon, leaving one end long. Repeat this, tying each piece of ribbon to an attachment lug. b) If the device has one attachment lug at the front and two at the back, cut the $120 \mathrm{~cm}$ ribbon into one $60 \mathrm{~cm}$ length and two $30 \mathrm{~cm}$ lengths. Tie each one of the $30 \mathrm{~cm}$ pieces of ribbon to one of the back-attachment lugs as above. With the longer $60 \mathrm{~cm}$ Teflon ribbon, feed this through the single attachment lug at the front of the device and pull this half the way through. Check that the lengths are equal and tie in a simple knot.

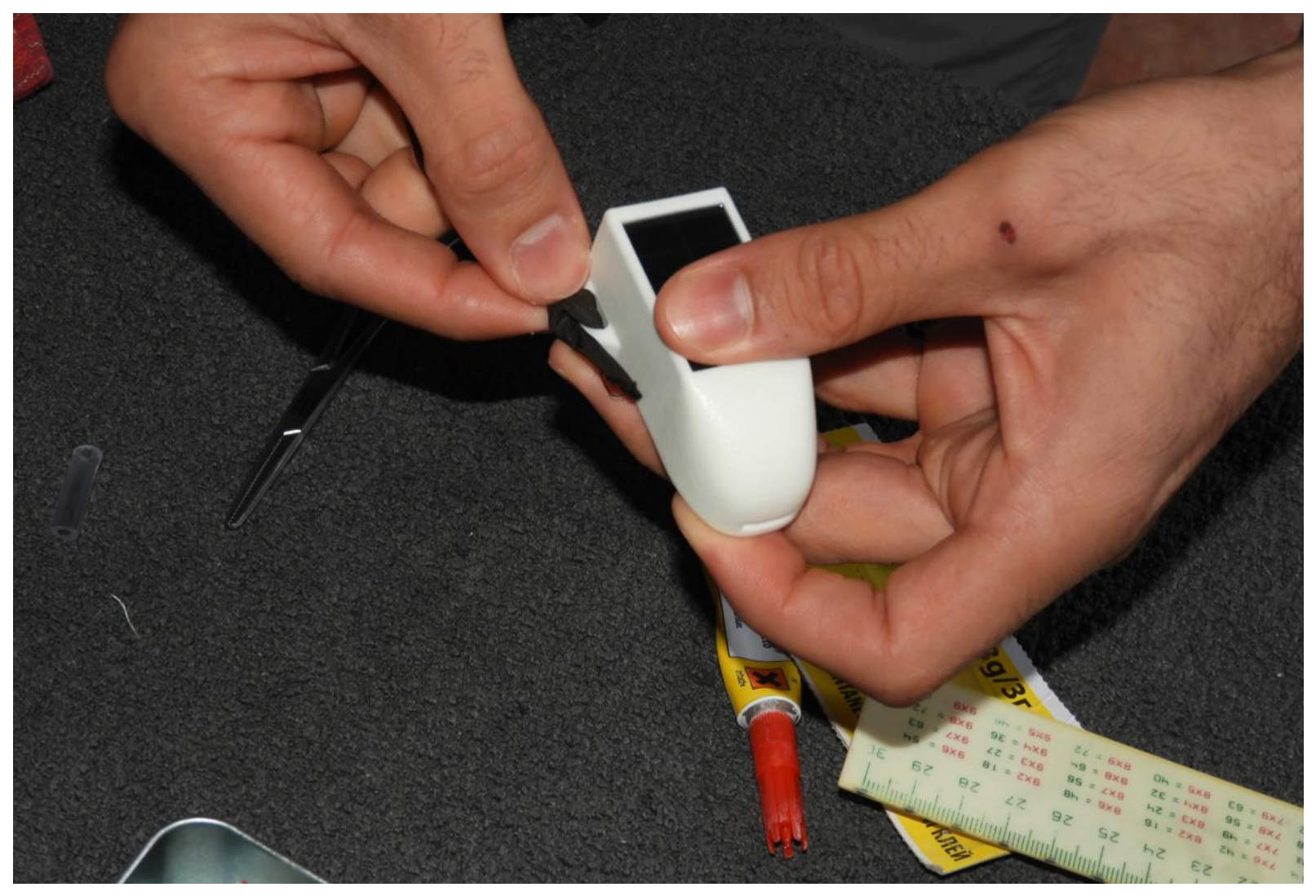

Figure 3.2: Feeding ribbon into the device. 


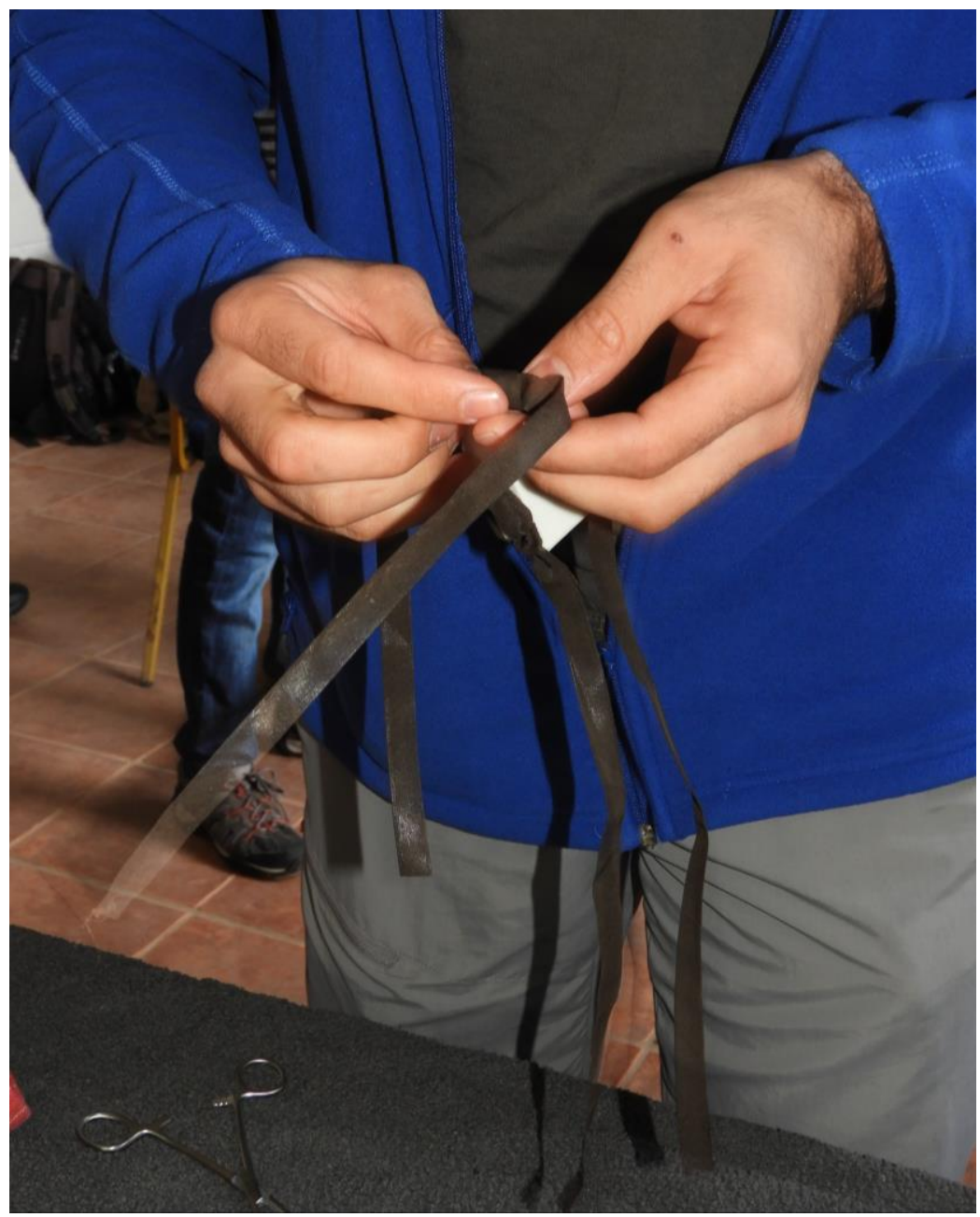

Figure 3.3: Tying the ribbon.

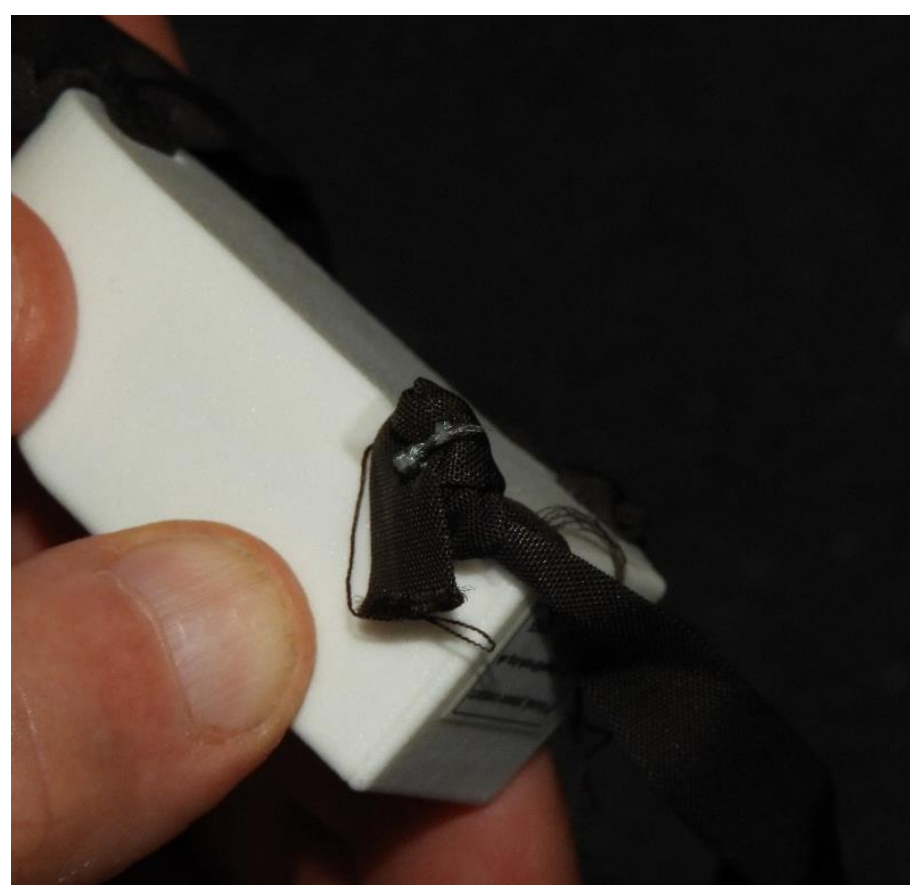

Figure 3.4: Knots on ribbon. 
2) Stitch through each knot using a needle and the Kevlar thread (a needle threader can be useful here). Tie a knot in the thread. Start sewing from the top downwards (towards the bird's body) so that the Kevlar knot sits on top. Sew three or four stitches into each knot. The end of the Teflon ribbon should be frayed and slightly unravelled. Incorporate the frayed ribbon into the last stitch

3) Apply superglue to the outward facing Kevlar/ribbon knots. This will help to keep the knot secure and to smooth down the edges.

4) Along each length of ribbon add scale marks using a ruler and a silver paint marker pen. From $15 \mathrm{~cm}$ from the device, make marks $10 \mathrm{~mm}$ apart. Number the lines on the ribbon. This will allow you to calculate how much ribbon makes up each strap of the harness and to ensure that the straps are of equal length. Write on same sides so that when attaching the device to the bird you can check that you have an even fit by matching up of the Kevlar knot. Then wrap the end thread around the needle three times to pull into a knot. Cut off the ends of thread.

If a three lugged device is being used, only two stitches are needed in the knot tied in the front lug with the $60 \mathrm{~cm}$ length of ribbon. This is because when fully attached there will be no loose ends of ribbon at this lug. the numbers.

5) On the underside of the device, use neoprene padding. Draw around the tag and cut the padding to fit (making sure you include a wider area to pad the undersides of the lugs and knots). Other than these wider parts of the neoprene under the lugs, the pad should fit the outline of the tag closely so that it does not fray or have excess areas of neoprene that the bird can pull at. Use neoprene glue to attach the pad to the bottom of the device.

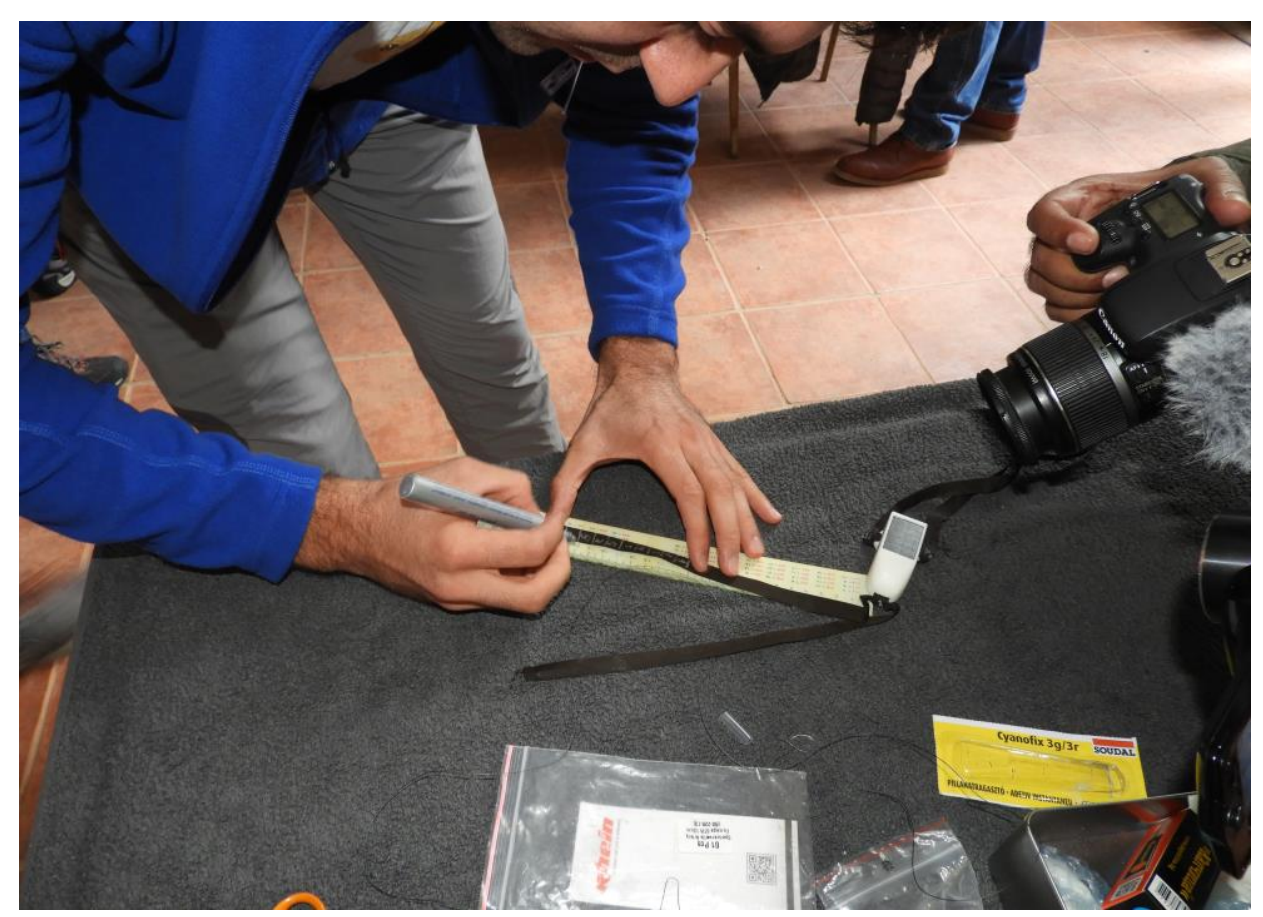

Figure 3.5: Marking the ribbon. 


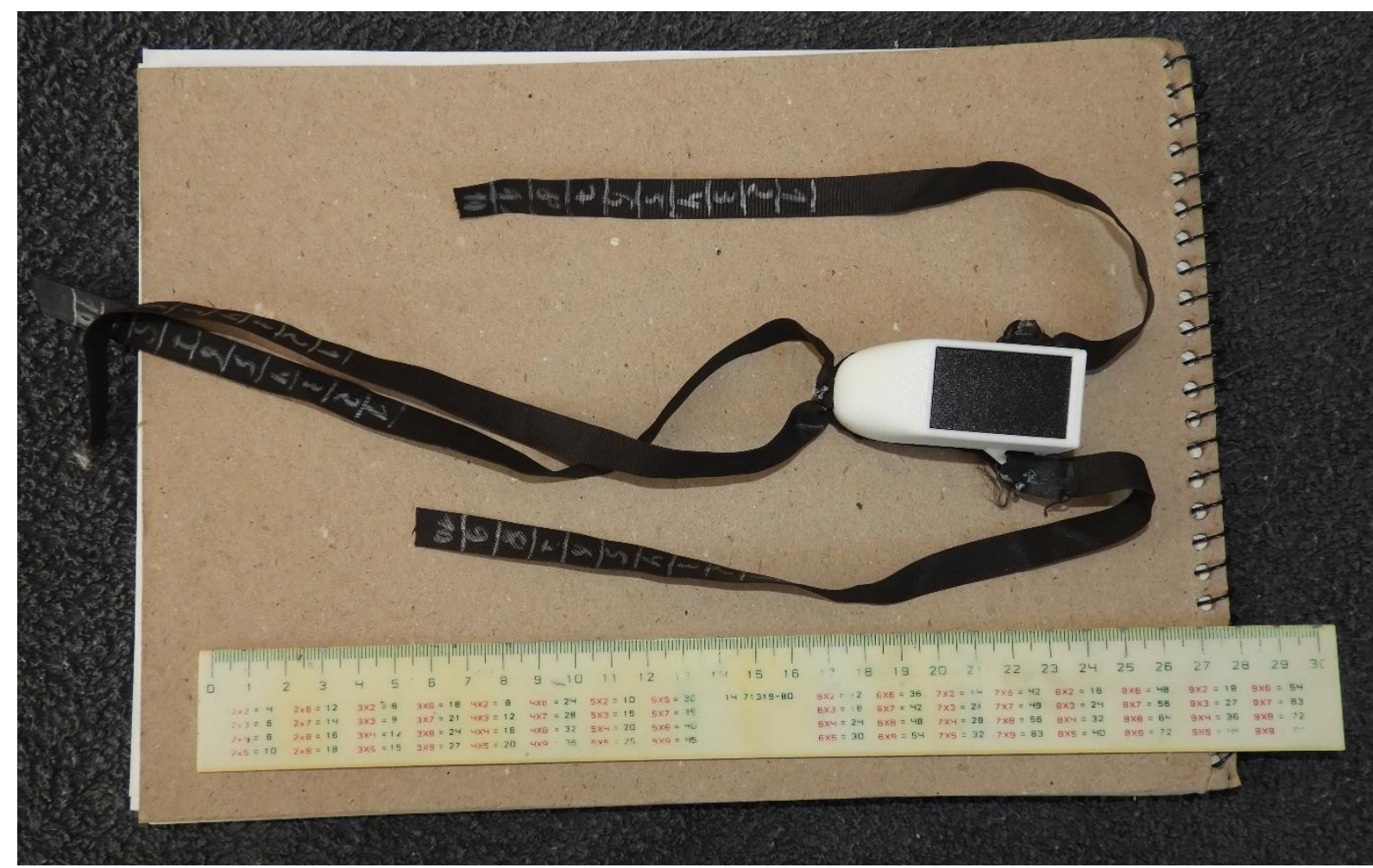

Figure 3.6: The prepared harness.

\section{Restraint and hooding}

In order to keep the bird calm while restrained, a loose material tube (or a sock with the toes cut off) can be used to cover the head. A hole in the end is needed so that the bird will not choke if it regurgitates food whilst hooded.

At this stage an additional person is needed to hold the bird whilst the tag is being attached. It is recommended that the bird is held breast downwards as much as possible, though angling the bird upwards will be necessary when making the final attachment at the sternum.

\section{Fitting to the bird}

1) The device should be positioned on the back in the approximate position where it will lie. Front straps can be fed around shoulders and back straps fed under the wings like a backpack.

2) The four straps then meet on the sternum and can be held in place using the clamp scissors.
Numbers from the top two straps and the bottom two straps should match up (e.g. two 4 s at the top, two $6 \mathrm{~s}$ at bottom). It is helpful to stand the bird on its feet (though still restrained) whilst assessing the fit.

3) Make sure the ribbons meet on the sternum and not over the crop. Adjust the feathering so that the straps are not forcing it from its natural position.

4) Assess the fit of the harness by putting two fingers between the tag and the bird's back. It should be possible to move the fingers but without there being a gap. It is better to leave the fit a little bit loose, in case the bird gains weight/grows, rather than having it too tight.

5) When satisfied, put a piece of card or cloth under the point where the ribbons join at the sternum to protect the bird. Then stitch the four ribbons together with Kevlar thread using the curved needle. Sew towards the bird's body so that all knots will be on the side of the harness away from the body. Stitch in each corner of the square where the ribbons meet, then remove the clamp. Cut 
off the loose ends of ribbon, then stitch two or three times in the centre of the square. The end of the Teflon ribbon should be frayed and slightly unravelled. Then incorporate the frayed ribbon into the last stitch of the Kevlar knot. Superglue the outward facing knots and the ribbon ends to achieve a smooth finish.

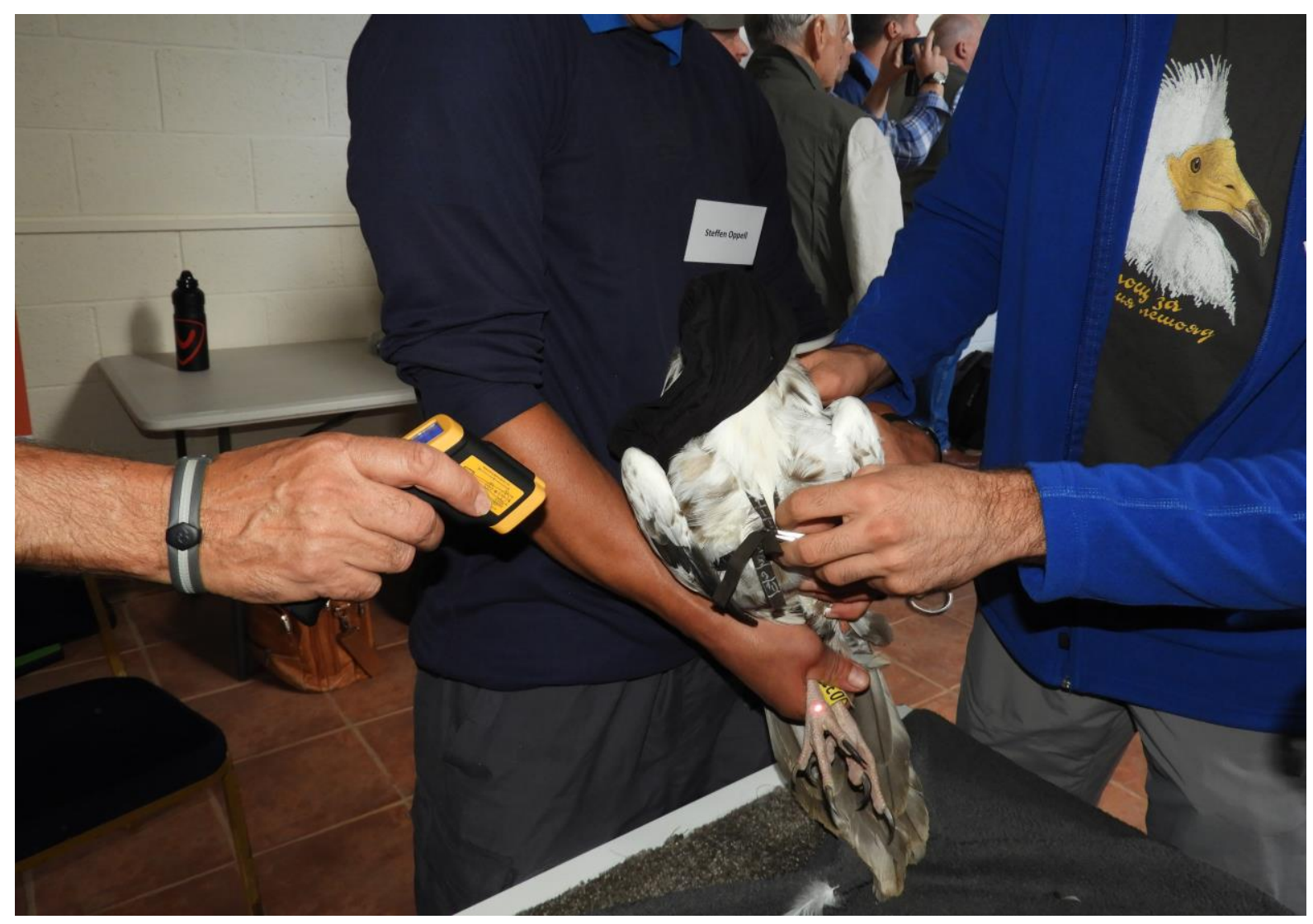

Figure 3.7: Assessing the fit. 